\title{
EDITORIAL
}

\section{Research in renewable energy materials: The fundamental physics and chemistry}

As the population of the world increases and people strive for a higher standard of living, the amount of energy necessary to sustain our society is ever increasing. While the supply of fossil fuels is very limited and they have had an adverse effect on the environment, it is imperative for all nations to explore the new energy sources which should be abundant, renewable, secure, clean, safe, and cost-effective. Critical to this goal is the research and development of materials for energy conversion and energy storage.

To get deeper insights into the fundamental physics and chemistry involved, in this special issue of Frontiers of Physics we invite a panel of experts to elucidate the state-of-the-art in the fundamental research of renewable energy materials. Prof. Feng Ding and coworker present the mechanisms, challenges and perspectives of hydrogen storage in physisorption, metal-assisted storage, chemisorption and spillover; Prof. Li-xian Sun and coworkers shed lights on thermodynamics and kinetics of new hydrogen storage materials; Prof. Qing Jiang and coworkers give an extensive review on density functional theory (DFT) calculations of the metal-doped carbon nanostructures as hydrogen storage materials; Prof. Fu-qiang Huang and coworkers address the major issues of solar materials for thin-film photovoltaic cells; Prof. Zhen Zhou and coworkers discuss the fundamental problems in designing new materials for $\mathrm{Li}$ ion batteries, and investigate hydrogen storage in $\mathrm{Sc}$ and $\mathrm{Ca}$ decorated BN nanosheets; Prof. Sa Li and coworkers study hydrogen storage and sensoring in Tidoped nano porous graphene; Prof. Li-min Wang and coworkers clarify the electrochemical hydrogen storage properties of melt-spun alloy; Prof. Ji-jun Zhao and coworkers investigate the substitution effects on the hydrogen storage behavior in $\mathrm{AB}_{2}$ alloys; Prof. Qiang Sun and coworkers design a tripyrrylmethane based 2D porous structure for hydrogen storage; and Prof. Hong Zhang and coworkers study the interactions of dihydrogen with lithium

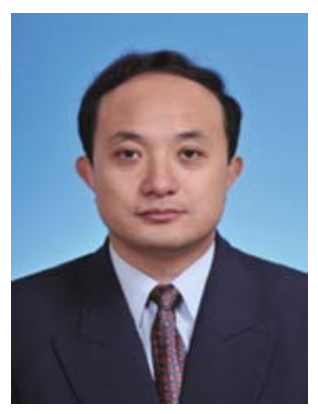

Prof. Jun-lin YANG

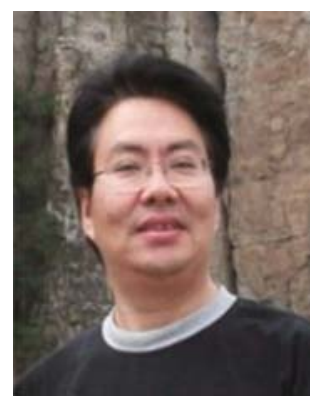

Prof. Qiang SUN containing organic complexes $\mathrm{C}_{4} \mathrm{H}_{4-m} \mathrm{Li}_{m}$ and $\mathrm{C}_{5} \mathrm{H}_{5-m} \mathrm{Li}_{m}(m=1,2)$ by means of density functional theory calculation. All the papers in the special issue are presented with greater rigor and at a high level of detail and strictly reviewed by referees. We hope that readers who are actively involved in research on renewable energy or want to gain depth in the field will find it beneficial.

We would like to thank all the contributors for their great effort. Our appreciations also go to Managing Editor Dong for his assistance.

Guest Editors

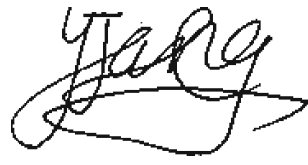

Professor Jun-lin YANG

National Natural Science Foundation of China

Beijing 100085, China

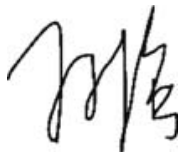

Professor Qiang SUN

College of Engineering

Peking University, Beijing 100871, China 